\title{
Leptomeningeal Carcinomatosis Presenting Early in Non-Small Cell Lung Cancer
}

\author{
Ezra N. Teitelbaum ${ }^{1}$, Susan M. Hasselquist ${ }^{2}$, Imad A. Tabbara ${ }^{3}$, Gregory D. Trachiotis ${ }^{{ }^{*}}$ \\ ${ }^{1}$ Division of Cardiothoracic Surgery, the George Washington University \\ Medical Center Department of Surgery, Washington DC, USA \\ ${ }^{2}$ Division of Hematology and Oncology, the George Washington University \\ Medical Center Department of Surgery, Washington DC, USA \\ ${ }^{3}$ Division of Pulmonary, Critical Care and Allergy, the George Washington University \\ Medical Center Department of Surgery, Washington DC, USA \\ E-mail: "gtrachiotis@mfa.gwu.edu \\ Received June 16, 2011; revised August 20, 2011; accepted September 22, 2011
}

\begin{abstract}
Purpose: we present the case of a patient presenting with altered mental status on post-operative day seven after a diagnostic lung biopsy. The diagnosis of carcinomatous letomeningeal metastasis was confirmed. Methods: we discuss meningeal carcinomatosis in lung cancer, and describe challenges to diagnosis, therapy, and prognosis. Results: a review of the literature describing the clinical and oncologic principles of letomeningeal carcinomatosis in lung cancer is performed. Discussion: leptomeningeal carcinomatosis (LC) occurs in approximately 5\% of patients with non-small cell lung cancer and incurs a bleak prognosis. Presenting neurologic symptoms can be varied and diagnosis is confirmed via lumbar puncture and cerebrospinal fluid cytology. Few data exist regarding optimal treatment, although intrathecal chemotherapy has been shown to provide a modest improvement in median survival.
\end{abstract}

Keywords: Leptomeningeal Carcinomatosis, Lung Cancer

\section{Introduction}

Leptomeningeal metastasis is an increasing complication of non-small cell lung cancer (NSCLC), occurring with an estimated incidence of approximately 5\% [1]. Widespread dissemination of tumor cells throughout the meninges and ventricles can also be seen in $5 \%$ of cases with adenocarcinoma of the breast, gastrointestinal tract, melanoma, childhood leukemia and systemic lymphoma [1-3]. Most often leptomenineal carcinomatosis (LC) presents in patients with cancer at the time when systemic disease has recurred or when prior chemotherapy regimens have failed. Thus, the goal of treatment for LC, if initiated, is often to control or preserve neurologic performance, while attempting to attenuate other widespread systemic disease and palliation. For NSCLC, the presentation of a neurologic process without brain parenchymal involvement early in the diagnosis is especially rare and unusual. This report details such a case, and describes current best modalities for diagnosis and palliative therapy.

\section{Case Report}

A 50 year old woman had a left upper lobe opacity found incidentally on a chest x-ray performed during a hospitalization for an anaphylactic reaction to a bee sting. The patient had no history of cough or shortness of breath, and no recent weight loss or bone pain, and did not smoke or have a family history of lung cancer. On physical examination the vital signs were normal, lungs were clear, neurologic exam was normal and there was no skeletal tenderness or palpable lymphadenopathy. A computed tomography (CT) scan of the chest revealed an isolated $2.2 \mathrm{~cm}$ spiculated lesion in the left upper lobe and no mediastinal adenoapthy (Figure 1). Positron emission tomography (PET) scan demonstrated hypermetabolic activity in the lung mass, as well as multiple hypermetabolic skeletal lesions in the left humerus, L1 and L3 vertebral bodies and right and left iliac bones all consistent with metastatic disease. A CT scan of the brain was normal without evidence of metastases.

Modalities of obtaining a tissue diagnosis, including 


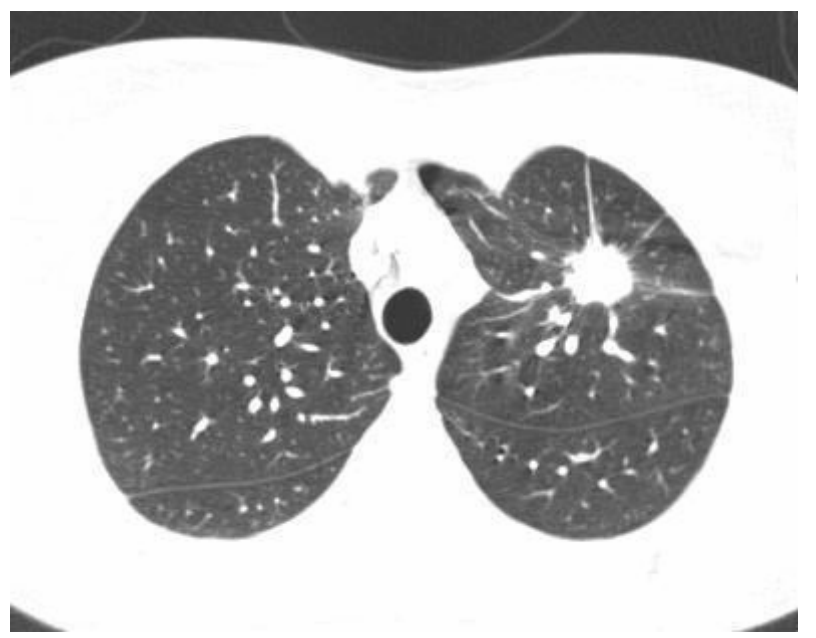

Figure 1. Chest CT showing the left upper lobe mass.

percutaneous biopsy of the lung or bony lesions or a surgical lung biopsy, were discussed and the patient opted to proceed with surgery. The patient had a left videoassisted thoracoscopic segmental resection of the left upper lobe mass, and pathology confirmed the mass to be a $2.2 \mathrm{~cm}$ moderately differentiated adenocarcinoma. Clinical, pathologic and radiographic studies classified the patient as Stage IV non-small cell lung cancer. Postoperatively the patient complained of an unusual amount of nausea and intense headaches. Her neurologic exam remained normal, a magnetic resonance imaging (MRI) of the brain obtained at this time was normal. Her symptoms improved with anti-emetics and a change in pain regimen and she was discharged home after chest tube removal on post-operative day three.

On post-operative day seven the patient was brought back to the emergency room after complaining of severe frontal headaches and then becoming increasingly somulent. On exam the patient had a temperature of 36.5, heart rate (HR) of 45, blood pressure (BP) of 155/80. She was drowsy and arousable to voice but not oriented and not able to follow commands. Her motor, sensory and cranial nerve exams were without focal deficits. A head CT revealed diffusely enlarged ventricles and an MRI showed increased meningeal enhancement in the posterior parietal and occipital lobes suggestive of meningitis (Figure 2). A lumbar puncture was performed with an opening pressure of $41 \mathrm{~cm}$. The drained cerebrospinal fluid (CSF) was clear with 9 leukocytes (90\% lymphocytes) and 2 erythrocytes per $\mu \mathrm{L}$. The patient was admitted to the intensive care unit and started on intravenous vancomycin, ceftriaxone and acyclovir. Her mental status temporarily improved but overnight she became obtunded with a HR of 38 and a BP of 175/80. A repeat head CT was unchanged and a lumbar drain was placed. With interval drainages of CSF, the patient's mental

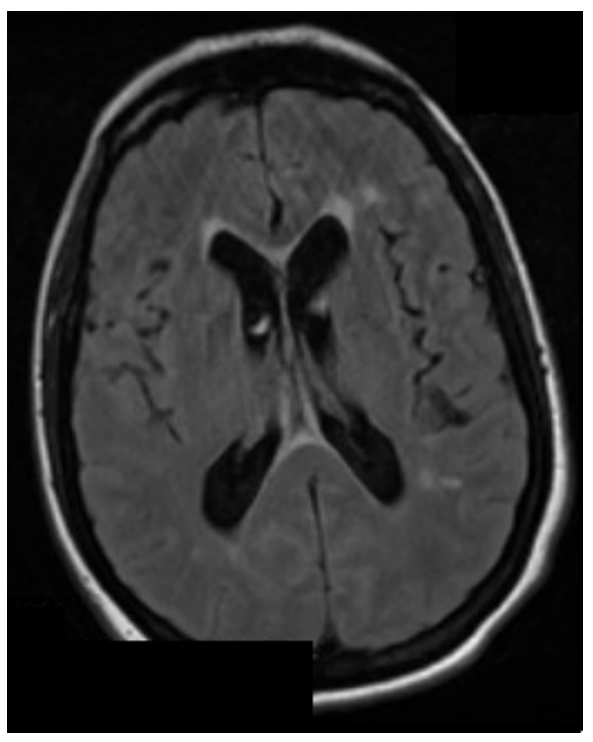

Figure 2. MRI with meninigeal enhancement in the parietal and occipital lobes.

status, HR and BP returned to normal. CSF cultures remained negative and cytology returned showing metastatic adenocarcinoma cells. Antibiotics were discontinued and the patient was started on intrathecal methotrexate through the lumbar drain. A ventricular reservoir and mediport were placed for further administration of intrathecal (thiopeta) and systemic chemotherapy (premetrexed and carboplatin), and she was discharged home. After the fourth cycle of systemic therapy, a repeat PET scan showed no change in the metastatic skeletal lesions. Four months after diagnosis she has lost 10 pounds and continues to have headaches but remains without other neurologic or respiratory symptoms.

\section{Discussion}

Of the $10 \%-15 \%$ of patients with neurologic metastatic lung cancer, approximately $5 \%$ of patients with nonsmall cell lung cancer will develop leptomenigeal carcinomatosis (LC) (or metastatic infiltration of the CSF). This is usually a later finding in patients who have already failed systemic chemotherapy regimens, although it can also be present at the time of initial diagnosis $[1,2]$.

Patients with leptomenigeal carcinomatosis (LC) can present with symptoms similar to infectious meningitis such as headache, somulence, neck stiffness, nausea and vomiting. Alternatively, direct nerve invasion can produce cranial nerve palsies or blood vessel infiltration can result in stroke-like patterns of extremity weakness [3].

CT scan of the head with intravenous contrast is used to rule out brain metastases and signs of increased intracranial pressure. MRI with gadolinium contrast can better evaluate for metastatic disease and detect signs of 
LC such as meningeal enhancement [3,4]. While imaging can serve as a useful guide, CSF histology remains the gold standard for diagnosis of LC. CSF cytology is positive for malignant cells in $50 \%-70 \%$ of cases, although this yield can be increased to $90 \%$ with second and third lumbar punctures. Increased opening pressures, high protein and low glucose levels are also common findings on CSF examination [5].

LC remains a bleak diagnosis with median survivals of one to five months in treated patients and four to six weeks if left untreated [2,6,7]. Wasserstrom reviewed 90 patients with LC from various primary tumors treated with intrathecal methotrexate. The 23 patients with all type lung cancer primaries had a 50\% response rate and a median survival of 4 months [6]. Chamberlain studied a selective population of patients with LC from non-small cell lung cancer primaries. These 32 patients were treated with intrathecal methotrexate and some received intrathecal cytarabine and thiotepa as second and third-line therapies respectively. Twelve of the patients received concurrent systemic chemotherapy. Complete cytological and partial clinical responses were seen in $43 \%$ of patients treated with methotrexate, $50 \%$ of those treated with cytarabine and $33 \%$ of those treated with thiotepa. Median survival was five months and no difference was seen in the patients who also received systemic chemotherapy [2]. Other therapies used in LC have included gemcitabine and ara-C, as well as radiotherapy and surgery to palliate compressive brain or spinal cord metastases [8].

\section{Conclusions}

LC is a rare complication of non-small cell lung cancer that imparts a dismal prognosis. Diagnosis is made by imaging and CSF evaluation with cytology. Treatment with intrathecal chemotherapy results in a small improvement in life expectancy. As such, palliation of symptoms and discussions with patients and their family members regarding realistic expectations for prognosis are important aspects of care.

\section{References}

[1] T. W. Shields, J. Locicero, C. E. Reed and R. H. Feins, "General Thoracic Surgery," 7th Edition, Williams \& Wilkins, Malvern, 2009.

[2] M. C. Chamberlain and P. Kormanik, "Carcinoma Meningitis Secondary to Non-small Cell Lung Cancer: Combined Modality Therapy," Archives of Neurology, Vol. 55, No. 4, 1998, pp. 506-512. doi:10.1001/archneur.55.4.506

[3] N. Pavlidis, "The Diagnostic and Therapeutic Management of Leptomeningeal Carcinomatosis," Annals of Oncology, Vol. 15, Supplement 4, 2004, pp. iv285-iv291. doi:10.1093/annonc/mdh941

[4] M. B. Fukui, C. C. Meltzer, E. Kanal and J. G. Smirniotopoulos, "MR Imaging of the Meninges," Radiology, Vol. 201, No. 3, 1996, pp. 605-612.

[5] E. T. Wong and J. T. Joseph, "Meningeal Carcinomatosis in Lung Cancer,” Journal of Clinical Oncology, Vol. 18, No. 15, 2000, pp. 2926-2927.

[6] W. Wasserstrom, J. Glass and J. Posner, “Diagnosis and Treatment of Leptomeningeal Metastases from Solid Tumors: Experience with 90 Patients,” Cancer, Vol. 49, No. 4, 1982, pp. 759-772. doi:10.1002/1097-0142(19820215)49:4<759::AID-CNC R2820490427>3.0.CO;2-7

[7] M. Balm and J. Hammack, "Leptomeningeal Carcinomatosis,” Archives of Neurology, Vol. 53, No. 7, 1996, pp. 626-632.

[8] M. Westphal, O. Heese and M. de Wit, "Intracranial Metastases: Therapeutic Options,” Annals of Oncology, Vol. 14, Supplement 3, 2003, pp. iii4-10. doi:10.1093/annonc/mdg741 